\title{
Solution to the Initial Value Problem for a High-Gain FEL via Van Kampen's Method
}

\author{
Zhirong Huang ${ }^{1}$, Kwang-Je Kim \\ Advanced Photon Source, Argonne National Laboratory, Argonne, IL 60439, USA
}

\begin{abstract}
Using Van Kampen's normal mode expansion, we solve the initial value problem for a high-gain free-electron laser (FEL) described by the three-dimensional MaxwellKlimontovich equations. An expression of the radiation spectrum is given for the process of coherent amplification and self-amplified spontaneous emission. It is noted that the input coupling coefficient for either process increases with the initial beam energy spread. The effective start-up noise is identified as the coherent fraction of the spontaneous undulator radiation in one field gain length, and is larger with increasing energy spread and emittance mainly because of the increase in gain length.
\end{abstract}

Key words: Initial value problem; High-gain free-electron laser; Self-amplified spontaneous emission

PACS: $41.60 . \mathrm{Cr} ; 42.55 . \mathrm{Vc}$

\section{Introduction}

The submitted manuscript has been $\mathrm{cr}$ by the University of Chicago as Opere Argonne National Laboratory ("Argo under Contract No. W-31-109-ENG-3i the U.S. Department of Energy. The Government retains for itself, and othe: ing on its behalf, a paid-up, nonexcl irrevocable worldwide license in said. to reproduce, prepare derivative work: tribute copies to the public, and perform ticly and display publicly, by or on bet the Government.

In a high-gain free-electron laser (FEL), a coherent external signal or the incoherent undulator radiation can initiate the FEL interaction to create an exponentially growing coherent radiation. Such a radiation is a promising source for future-generation x-ray facilities. Thus, it is important to understand how the exponential process starts and how the incoherent radiation develops into a coherent source.

1 Corresponding author. Tel: (630)252-6023; Fax: (630)252-5703; Email: zrh@aps.anl.gor 


\section{DISCLAIMER}

This report was prepared as an account of work sponsored by an agency of the United States Government. Neither the United States Government nor any agency thereof, nor any of their employees, make any warranty, express or implied, or assumes any legal liability or responsibility for the accuracy, completeness, or usefulness of any information, apparatus, product, or process disclosed, or represents that its use would not infringe privately owned rights. Reference herein to any specific commercial product, process, or service by trade name, trademark, manufacturer, or otherwise does not necessarily constitute or imply its endorsement, recommendation, or favoring by the United States Government or any agency thereof. The views and opinions of authors expressed herein do not necessarily state or reflect those of the United States Government or any agency thereof. 


\section{DISCLAIMER}

Portions of this document may be illegible in electronic image products. Images are produced from the best available original document. 
The FEL initial value problem was solved in Ref. $[1,2]$ using a one-dimensional (1-D) theory. The three-dimensional (3-D) initial value problem for parallel beam was studied by Van Kampen's method in Ref. [3,4] and by a Green's function technique in Ref. $[5,6]$. Recently, Xie [7] studied the initial value problem including emittance and found that the effective start-up noise in self-amplified spontaneous emission (SASE) becomes significantly larger with finite emittance and energy spread.

Inspired by the work of Xie, we explain the solution to the FEL initial value problem using Van Kampen's method applicable to the 3-D case including emittance. We then attempt to provide an understanding of the dependence of the effective start-up noise on beam parameters. Two factors determining the start-up process are identified. The input coupling coefficient for both coherent amplification (CA) and SASE is found to increase with the initial energy spread. The effective start-up noise is shown to be the coherent fraction of the spontaneous undulator radiation in the first field gain length, generalizing the result of Ref. [6] for a beam with vanishing energy spread and emittance. The effective start-up noise appears to be larger with increasing energy spread and emittance mainly because of the increase of the gain length.

\section{The Dynamic Equations and the Initial Conditions}

It is convenient to use $\bar{z}=2 \rho k_{u} z$, the scaled distance from the undulator entrance, as the independent "time" variable. Here $\rho \ll 1$ is the FEL parameter [8], $k_{u}=2 \pi / \lambda_{u}$, and $\lambda_{u}$ is the undulator period. The scaled transverse phase-space variables are $\bar{x}=x \sqrt{2 k_{1} k_{u} \rho}$ and $\bar{p}=p \sqrt{k_{1} /\left(2 k_{u} \rho\right)}$, where $x=(x, y) \cdot p=(d x / d z, d y / d z)$. and $c k_{1}=2 \pi c / \lambda_{1}$ is the fundamental radiation frequencr. The longitudinal variables are the relative bunch position $\theta$ in 
units of $2 \pi / \lambda_{1}$ and the scaled energy deviation $\vec{\eta}=\left(\gamma-\gamma_{0}\right) /\left(\rho \gamma_{0}\right)$. To take into account the discreteness of the electron beam, we employ the Klimontovich distribution function [9]:

$$
f(\theta, \bar{\eta}, \overline{\boldsymbol{x}}, \overline{\boldsymbol{p}} ; z)=\frac{2 k_{1}^{2} k_{u} \rho}{n_{0}} \sum_{j=1}^{N_{e}} \delta\left(\theta-\theta_{j}\right) \delta\left(\bar{\eta}-\bar{\eta}_{j}\right) \delta\left(\overline{\boldsymbol{x}}-\tilde{\boldsymbol{x}}_{j}\right) \delta\left(\overline{\boldsymbol{p}}-\overline{\boldsymbol{p}}_{j}\right)
$$

where $n_{0}$ is the peak electron volume density, and $N_{e}$ is the total number of electrons. Following Ref. [10], the fundamental electric field $a_{\nu}$ is represented by

$$
a_{\nu}(\overline{\boldsymbol{x}} ; \bar{z})=\frac{-e K[\mathrm{JJ}]}{4 \gamma_{0}^{2} m c^{2} k_{u} \rho} e^{-i \overline{\bar{\nu}} \bar{z}} E_{\nu}(\overline{\boldsymbol{x}} ; \bar{z})
$$

where $K$ is the undulator parameter, $[\mathrm{JJ}]=J_{0}(\xi)-J_{1}(\xi)\left(\xi=K^{2} /\left(4+2 K^{2}\right)\right)$, $E_{\nu}$ is the complex field amplitude at frequency $\omega=\nu c k_{1}$, and $\bar{\nu}=(\nu-1) /(2 \rho)$ is the scaled frequency detuning.

In the small signal regime before saturation, we write $f(z)=f_{0}+f_{1}(z)$, where $f_{0}$ is the initial smooth background distribution, and $f_{1}(z)$ contains the initial fluctuation as well as the effects of the FEL interaction and is also treated as a small quantity. Here we assume that the initial electron beam is matched to the undulator channel and is uniform in $\theta$ (this can be approximately satisfied if the bunch length $\theta_{b} \gg 1$ ). We introduce the state vector

$$
\Phi(z)=\left(\begin{array}{c}
a_{\nu}(\overline{\boldsymbol{x}} ; \bar{z}) \\
f_{\nu}(\bar{\eta}, \overline{\boldsymbol{x}}, \overline{\boldsymbol{p}} ; \bar{z}) \equiv \int \frac{2 \rho d \theta}{2 \pi} e^{-i \nu \theta} f_{1}(\theta, \bar{\eta}, \overline{\boldsymbol{x}}, \overline{\boldsymbol{p}} ; \bar{z})
\end{array}\right),
$$

and define the scalar product $\left(\Phi, \Phi^{\prime}\right) \equiv \int d^{2} \bar{x} a_{\nu} a_{\nu}^{\prime}+\int d^{2} \bar{x} \int d^{2} \bar{p} \int d \bar{\eta} f_{\nu} f_{\nu}^{\prime}$. The Maxwell-Klimontovich equations can be combined as [10]

$$
\left(\frac{\partial}{\partial \bar{z}}-i \mathrm{M}\right) \Phi=0
$$


Here the operator $M$ is

$$
\operatorname{M} \Phi(\bar{z})=\left(\begin{array}{c}
\left(-\bar{\nu}+\frac{\bar{\nabla}_{\perp}^{2}}{2}\right) a_{\nu}-i \int d^{2} \bar{p} \int d \bar{\eta} f_{\nu} \\
-i a_{\nu} \frac{\partial f_{0}}{\partial \bar{\eta}}+\left[-\nu\left(\bar{\eta}-\frac{1}{2}\left(\bar{p}^{2}+\bar{k}^{2} \bar{x}^{2}\right)\right)+i\left(\bar{p} \frac{\partial}{\partial \bar{x}}-\bar{k}_{\beta}^{2} \bar{x} \frac{\partial}{\partial \bar{p}}\right)\right] f_{\nu}
\end{array}\right)
$$

where $\vec{\nabla}_{\perp}=\partial /(\partial \bar{x})$ is the scaled transverse Laplacian, $\vec{k}_{\beta}=k_{\beta} /\left(2 k_{u} \rho\right)$ is the scaled transverse focusing strength, and $\bar{k}=K /\left(4 \gamma_{0} \rho\right)$ is the scaled natural focusing strength.

The evolution of the radiation field and the distribution function in the startup and the exponential growth regimes is completely determined by Eq. (4) and the initial value $\Phi(0)$ of the state vector. The latter is specified by the

external signal $a_{\nu}(0)$ and the shot noise $f_{\nu}(0)=\int \frac{2 \rho d \theta}{2 \pi} e^{-i \nu \theta} f_{1}(0)$. Although the ensemble average of $f_{\nu}(0)$ is zero, physically meaningful quantities such as intensity can be computed by using the relation [9]:

$$
\left\langle f_{\nu}(\bar{\eta}, \overline{\boldsymbol{x}}, \overline{\boldsymbol{p}} ; 0) f_{\nu}\left(\bar{\eta}^{\prime}, \overline{\boldsymbol{x}}^{\prime}, \overline{\boldsymbol{p}}^{\prime} ; 0\right)\right\rangle=\frac{2 k_{1}^{2} k_{u} \rho \theta_{b}}{n_{0}}\left(\frac{2 \rho}{2 \pi}\right)^{2} \delta\left(\bar{\eta}-\bar{\eta}^{\prime}\right) \delta\left(\overline{\boldsymbol{x}}-\overline{\boldsymbol{x}}^{\prime}\right) \delta\left(\overline{\boldsymbol{p}}-\overline{\boldsymbol{p}}^{\prime}\right) f_{0} .
$$

\section{Van Kampen's Normal Mode Expansion}

The initial value problem formulated in the previous section can be solved by expanding the solution in terms of the eigenvectors of Eq. (4). The coefficients of the expansion are easily determined from the initial conditions if the eigenvectors are mutually orthogonal under a suitably defined scalar product. The procedure is well-known in quantum mechanics in which all operators are Hermitian. Here $\mathbf{M}$ is not a Hermitian operator, and we employ the extension

of the method developed by Van Kampen [11] in studying the 1-D plasma waves. 
Let us first find the eigenvalues and the eigenvectors of Eq. (4), defined to be solutions

$$
e^{-i \mu_{n} \bar{z}} \Psi_{n}=e^{-i \mu_{n} \bar{z}}\left(\begin{array}{c}
A_{n}(\overline{\boldsymbol{x}}) \\
F_{n}(\bar{\eta}, \overline{\boldsymbol{x}}, \overline{\boldsymbol{p}})
\end{array}\right) .
$$

Solving the eigenvalue equation $\left(\mu_{n}+\mathbf{M}\right) \Psi_{n}=0$, we obtain

$$
F_{n}=\frac{\partial f_{0}}{\partial \bar{\eta}} \int_{-\infty}^{0} d \tau A_{n}\left(\bar{x}_{\beta}(\tau)\right) e^{i \phi_{\beta}(\tau)}
$$

and the mode equation

$$
\left(-i \mu_{n}+i \bar{\nu}+\frac{\bar{\nabla}_{\perp}^{2}}{2 i}\right) A_{n}(\overline{\boldsymbol{x}})-\int d^{2} \bar{p} \int d \bar{\eta} \int_{-\infty}^{0} d \tau A_{n}\left(\overline{\boldsymbol{x}}_{\beta}(\tau)\right) e^{i \phi_{\beta}(\tau)} \frac{\partial f_{0}}{\partial \bar{\eta}}=0,
$$

where $\overline{\boldsymbol{x}}_{\beta}(\tau)=\overline{\boldsymbol{x}} \cos \bar{k}_{3} \tau+\frac{\bar{p}}{k_{3}} \sin \bar{k}_{\beta} \tau$, and

$$
\begin{aligned}
\phi_{\beta}(\tau)= & {\left[\bar{\eta}-\frac{1}{4}\left(1+\frac{\bar{k}^{2}}{\bar{k}_{\beta}^{2}}\right)\left(\bar{p}^{2}+\bar{k}_{\beta}^{2} \bar{x}^{2}\right)-\mu_{n}\right] \tau } \\
& +\frac{1}{8}\left(1-\frac{\bar{k}^{2}}{\bar{k}_{3}^{2}}\right)\left[\left(\bar{k}_{\beta}^{2} \bar{x}^{2}-\bar{p}^{2}\right) \frac{\sin 2 \bar{k}_{\beta} \tau}{\bar{k}_{\beta}}+2 \overline{\boldsymbol{x}} \cdot \overline{\boldsymbol{p}}\left(1-\cos 2 \bar{k}_{\beta} \tau\right)\right] .
\end{aligned}
$$

Equation (9) is the dispersion relation derived in Ref. [12], which generalizes the result of Ref. $[3,13]$ to include the external focusing. It can be solved using a variational principle $[13,12,14]$ and a matrix formalism [14]. In general, a discrete set of eigenvalues and eigenmodes exists.

To define the Van Kampen orthogonality of these eigenvectors, we introduce the adjoint eigenvalue equation $\left(\tilde{\mu}_{n}+\tilde{\mathrm{M}}\right) \tilde{\Psi}_{n}=0$, where $\tilde{\mu}_{n}$ and $\tilde{\Psi}_{n}=\left(\tilde{A}_{n}, \tilde{F}_{n}\right)$ are the adjoint eigenvalues and eigenvectors of the adjoint operator $\tilde{\mathbf{M}}$, defined through

$$
\left(\tilde{\mathrm{M}} \tilde{\Psi}_{n}, \Phi\right)=\left(\tilde{\Psi}_{n}, \mathrm{M} \Phi\right)
$$

It is straightforward to show that $\tilde{F}_{n}=\int_{-\infty}^{0} d \tau \tilde{A}_{n}\left(\bar{x}_{3}(\tau)\right) e^{i \phi_{3}(\tau)}$ and that $\tilde{A}_{n}$ satisfies the same mode equation as Eq. (9). Hence we set $\tilde{A}_{n}=\tilde{A}_{n}$ and 
$\tilde{\mu}_{n}=\mu_{n}$.

By virtue of Eq. (11), one immediately obtains

$$
\left(\mu_{n}-\mu_{m}\right)\left(\tilde{\Psi}_{m}, \Psi_{n}\right)=\left(\tilde{\Psi}_{m}, \mathbf{M} \Psi_{n}\right)-\left(\tilde{\mathbf{M}} \tilde{\Psi}_{m}, \Psi_{n}\right)=0
$$

If these normal modes are not degenerate, i.e., $\mu_{n} \neq \mu_{m}$ for any $n \neq m$, the Van Kampen orthogonality for a discrete set of eigenvectors follows [11]:

$$
\left(\tilde{\Psi}_{m}, \Psi_{n}\right)=\delta_{m, n}\left(\tilde{\Psi}_{n}, \Psi_{n}\right) \equiv \delta_{m, n} C_{n}
$$

where $C_{n}$ is the normalization for the $n^{\text {th }}$ mode and is given by

$$
C_{n}=\int d^{2} \bar{x} A_{n}^{2}(\overline{\boldsymbol{x}})+\int d^{2} \bar{x} \int d^{2} \bar{p} \int d \bar{\eta} \frac{\partial f_{0}}{\partial \bar{\eta}}\left[\int_{-\infty}^{0} d \tau A_{n}\left(\overline{\boldsymbol{x}}_{\beta}(\tau)\right) e^{i \phi_{\beta}(\tau)}\right]^{2} .
$$

In the case of degeneracy, an orthogonal set can be constructed through linear combinations of eigenvectors. For a continuous set of eigenvalues and eigenvectors, one replaces the Kronecker delta function $\delta_{m, n}$ in Eq. (13) with the Dirac delta function [11]. Furthermore, assuming the set of eigenvectors is complete, we obtain the formal solution for

$$
\Phi(\bar{z})=\sum_{n} \frac{\left(\tilde{\Psi}_{n}, \Phi(0)\right)}{C_{n}} \Psi_{n} e^{-i \mu_{n} \bar{z}}
$$

Equation (15) can be simplified significantly in the high-gain limit, where the fundamental mode $A_{0}(\bar{x})$ dominates because its eigenvalue $\mu_{0}$ has the largest imaginary part $\mu_{I}$. Dropping all other modes in the summation of Eq. (15), we obtain the evolution of the electric field in the high-gain regime $[10,7]$

$$
\begin{aligned}
a_{\nu}(\overline{\boldsymbol{x}} ; \bar{z})=\frac{1}{C_{0}} A_{0}(\overline{\boldsymbol{x}}) e^{-i \mu_{0} \bar{z}}[ & \int d^{2} \bar{x}^{\prime} \cdot A_{0}\left(\overline{\boldsymbol{x}}^{\prime}\right) a_{\nu}\left(\overline{\boldsymbol{x}}^{\prime} ; 0\right)+\int d^{2} \bar{x}^{\prime} \int d^{2} \bar{p} \int d \bar{\eta} \\
& \left.\times f_{\nu}\left(\bar{\eta} \cdot \overline{\boldsymbol{x}}^{\prime}, \overline{\boldsymbol{p}} ; 0\right) \int_{-\infty}^{0} d \tau A_{0}\left(\overline{\boldsymbol{x}}_{3}(\tau)\right) e^{i \phi_{3}(\tau)}\right] .
\end{aligned}
$$

The first term in the square bracket describes the process of coherent amplification, which starts from an external signal $a_{\nu}(0)$. The second term describes 
the process of self-amplified spontaneous emission, which starts from white noise. Equation (16) for the parallel e-beam (with vanishing emittance) reduces to those of Ref. $[3,5]$. The ensemble averaged spectrum of the radiation intensity (power per unit area) can be computed with Eq. (6):

$$
\begin{aligned}
& \frac{1}{\rho I_{\text {beam }}} \frac{d I}{d \nu}=\frac{2 \pi}{(2 \rho)^{2} \theta_{b}}\left\langle\left|a_{\nu}(\overline{\boldsymbol{x}} ; z)\right|^{2}\right\rangle \\
& =\frac{1}{\left|C_{0}\right|^{2}}\left|A_{0}(\overline{\boldsymbol{x}})\right|^{2} e^{2 \mu_{I} \bar{z}}\left[\frac{2 \pi}{(2 \rho)^{2} \theta_{b}}\left|\int d^{2} \bar{x}^{\prime} A_{0}\left(\overline{\boldsymbol{x}}^{\prime}\right) a_{\nu}\left(\overline{\boldsymbol{x}}^{\prime} ; 0\right)\right|^{2}\right. \\
& \left.+\frac{2 k_{1}^{2} k_{u} \rho}{2 \pi n_{0}} \int d^{2} \bar{x}^{\prime} \int d^{2} \bar{p} \int d \bar{\eta} f_{0}\left(\bar{\eta}, \overline{\boldsymbol{x}}^{\prime}, \overline{\boldsymbol{p}}\right)\left|\int_{-\infty}^{0} d \tau A_{0}\left(\overline{\boldsymbol{x}}_{\beta}(\tau)\right) e^{i \phi_{\beta}(\tau)}\right|^{2}\right]
\end{aligned}
$$

where $I_{\text {beam }}=\gamma_{0} m c^{3} n_{0}$.

\section{Effects of Energy Spread}

To isolate the energy spread effects in the FEL start-up process, we look at the $1-\mathrm{D}$ limit of the above results by setting $A_{n}(\overline{\boldsymbol{x}})=1, \int d^{2} \tilde{x}=2 k_{1} k_{u} \rho \Sigma(\Sigma$ is the beam cross section) and dropping $\int d^{2} \vec{p}$ and the transverse Laplacian. The mode Eq. (9) reduces to the 1-D dispersion relation [1]:

$$
D(\mu)=\mu-\bar{\nu}-\int d \bar{\eta} \frac{d V / d \bar{\eta}}{\bar{\eta}-\mu}=0
$$

where $f_{0}=V(\bar{\eta})$ with $\int d \bar{\eta} V(\bar{\eta})=1$. For a monoenergetic beam (i.e., $V(\bar{\eta})=$ $\delta(\bar{\eta})$ ), this reduces to the cubic equation [8] with a growing, a decaying and an oscillatory solution. The intensity spectrum of Eq. (17) becomes the power spectrum of Ref. [1]:

$$
\frac{d P}{d \omega}=\frac{d I}{d \nu} \frac{\Sigma}{c k_{1}}=g_{A} e^{2 \mu_{I} \bar{z}}\left[\left(\frac{d P}{d \omega}\right)_{C}+\left(\frac{d P}{d \omega}\right)_{S}\right],
$$


where

$$
\begin{aligned}
g_{A} & =\left[1-2 \int d \bar{\eta} \frac{V(\bar{\eta})}{(\bar{\eta}-\mu)^{3}}\right]^{-2}=\left(\frac{d D}{d \mu}\right)^{-2} \\
\left(\frac{d P}{d \omega}\right)_{C} & =\frac{\pi P_{\text {beam }}}{2 \rho c k_{1} \theta_{b}}\left|a_{\nu}(0)\right|^{2} \quad \text { (coherent input power), } \\
\left(\frac{d P}{d \omega}\right)_{S} & =g_{S} \frac{\rho \gamma_{0} m c^{2}}{2 \pi} \quad \text { (effective start-up noise) } \\
g_{S} & =\int d \bar{\eta} \frac{V(\bar{\eta})}{|\bar{\eta}-\mu|^{2}}=\int d \bar{\eta} \frac{V(\bar{\eta})}{\mu_{I}^{2}+\left(\bar{\eta}-\mu_{R}\right)^{2}}
\end{aligned}
$$

Here $P_{\text {beam }}=I_{\text {beam }} \Sigma$ is the beam power, and $\mu=\mu_{R}+i \mu_{I}$ is a function of the frequency detuning $\bar{v}$ through the dispersion relation. For CA, the amplification occurs at the frequency defined by the frequency of the coherent input signal. For SASE, the frequency dependence is determined by $\mu_{I}(\bar{\nu})$ in the exponent of Eq. (19). Thus, $g_{A}$ and $g_{S}$, evaluated at the optimal detuning $\bar{\nu}_{0}$ where the growth rate $\mu_{I}$ reaches the maximum, determine the input coupling to the exponentially growing mode and the effective noise power in units of $\rho \gamma_{0} m c^{2} /(2 \pi)$, respectively.

In Ref. [1], $G=g_{A} g_{S}$ has been computed numerically for a flat-top energy distribution and has been found to increase initially with energy spread. For a Gaussian energy distribution

$$
V(\bar{\eta})=\frac{1}{\sqrt{2 \pi} \bar{\sigma}_{\eta}} \exp \left(-\frac{\bar{\eta}^{2}}{2 \bar{\sigma}_{\eta}^{2}}\right)
$$

we compute $\mu_{I}, g_{A}$ and $g_{S}$ (Fig. 1) as functions of the rms energy spread $\bar{\sigma}_{\eta}=$ $\sigma_{\eta} / \rho$ and find that both $g_{A}$ and $g_{S}$ increase with $\bar{\sigma}_{\eta}$. For a monoenergetic beam, any initial signal (external or spontaneous) couples equally well to the three (growing, decaying and oscillatory) modes that have the same normalization factor, hence we have the well-known $g_{A}=1 / 9$. However, $g_{A}$ is larger for a larger energy spread. approaching $1 / 4$ for the flat-top model and 1 for the Gaussian model. This implies the input coupling to the exponentially growing 
mode is preferred in the presence of energy spread for both CA and SASE.

The increase in the effective start-up noise through $g_{S}$ may be interpreted in the following way. First of all, $g_{S}=1$ for a monoenergetic beam, and the quantity $\rho \gamma_{0} m c^{2} /(2 \pi)$ is approximately the noise power generated from the spontaneous undulator radiation in the first field gain length $L_{g 0}=1 /\left(k_{u} \rho \sqrt{3}\right)[6]$. For a beam with a finite energy spread, the spontaneous radiation spectrum in the forward direction is the convolution of the beam energy spectrum and the undulator radiation spectrum with an intrinsic bandwidth $2 \Delta \eta=\Delta \nu=$ $\Delta \omega / \omega \sim 2 \pi /\left(k_{u} z\right):$

$$
\left(\frac{d P}{d \omega}\right)_{z}^{\text {spont }}=\frac{\lambda_{1}^{2}}{\Sigma}\left(\frac{d P}{d \omega d \Omega}\right)_{z}^{\text {spont }} \int d \eta V(\eta) S_{u}\left(k_{u} z(\eta-\Delta \nu / 2)\right),
$$

where $S_{u}(x)=\sin ^{2}(x) / x^{2}$ and

$$
\left(\frac{d P}{d \omega d \Omega}\right)_{z}^{\text {spont }}=\frac{\gamma_{0}^{2} m c^{2}}{4 \pi^{2}} \frac{I_{e}}{I_{A}}\left(\frac{K[\mathrm{JJ}]}{1+K^{2} / 2}\right)^{2}\left(k_{u} z\right)^{2}
$$

is the angular power spectrum with vanishing energy spread [15], $I_{e}$ is the electron current, and $I_{A}=17045 \mathrm{~A}$ is the Alfven current. After the first field gain length $z=L_{g}=\left(2 k_{u} \rho \mu_{I}\right)^{-1}$, we have

$$
\left(\frac{d P}{d \omega}\right)_{L_{g}}^{\text {spont }}=\frac{\rho \gamma_{0} m c^{2}}{2 \pi \mu_{I}^{2}} \int d \bar{\eta} V(\bar{\eta}) S_{u}\left(\frac{\bar{\eta}-\bar{\nu}}{2 \mu_{I}}\right) .
$$

Here we have used the relation

$$
\rho=\left[\frac{1}{8 \pi} \frac{\lambda_{1}^{2}}{\Sigma} \frac{I_{e}}{I_{A}}\left(\frac{K[\mathrm{JJ}]}{1+K^{2} / 2}\right)^{2}\right]^{1 / 3} .
$$

Comparing Eq. (22) in the form

$$
\left(\frac{d P}{d \omega}\right)_{S}=\frac{\rho \gamma_{0} m c^{2}}{2 \pi \mu_{I}^{2}} \int d \bar{\eta} \Gamma^{-}(\bar{\eta})\left[\frac{1}{1+\left(\bar{\eta}-\mu_{R}\right)^{2} / \mu_{I}^{2}}\right]
$$

with $\mathrm{Eq} .(27)$. we may interpret the effective start-up noise as the fraction of the spontaneous undulator radiation in the first field gain length within 
the coherent gain bandwidth $\Delta \bar{\eta} \sim \Delta \bar{\nu} \sim \mu_{I}$ (much narrower than the intrinsic undulator bandwidth $\left.2 \pi \mu_{I}\right)$. Using the Gaussian energy distribution in Eq. (24) and approximating the Lorentzian in the square bracket of Eq. (29) by another Gaussian, we can carry out the $\bar{\eta}$-integral to obtain

$$
\left(\frac{d P}{d \omega}\right)_{S} \approx \frac{\rho \gamma_{0} m c^{2}}{2 \pi \mu_{I}^{2}} \exp \left(-\frac{\mu_{R}^{2}}{2 \bar{\sigma}_{\eta}^{2}+\mu_{I}^{2}}\right) \frac{1}{\sqrt{1+2 \bar{\sigma}_{\eta}^{2} / \mu_{I}^{2}}}
$$

With increasing energy spread, the coherent fraction of the spontaneous radiation decreases, but the drop in the growth rate significantly increases the spontaneous radiation power in one field gain length, leading to the overall increase of the effective noise power through $g_{S}$ (as seen in Fig. 1). In fact, for large values of the energy spread $\bar{\sigma}_{\eta}^{2} \gg 1, \mu_{R} \approx \bar{\nu}_{0} \approx-\bar{\sigma}_{\eta}$ and $\mu_{I} \approx 0.76 / \bar{\sigma}_{\eta}^{2}[16]$, so that $(d P / d \omega)_{S} \propto \bar{\sigma}_{\eta}$ increases without bound because the effective noise power required to start the SASE process is infinite!

\section{Effects of Emittance}

We now return to the full 3-D Eq. (17) and consider SASE (the second term) only. Assuming that the betatron oscillations are slow on the scale of the gain length, we take $\bar{k}_{\beta} \tau \ll 1, \bar{x}_{\beta}^{\prime}(\tau) \approx \bar{x}^{\prime}$,

$$
\phi_{3}(\tau) \approx\left[\bar{\eta}-\frac{1}{2}\left(\overline{\boldsymbol{p}}^{2}+\bar{k}^{2} \overline{\boldsymbol{x}}^{2}\right)-\mu_{0}\right] \tau
$$

and integrate $\int d x^{2}(d I / d \omega)$ to obtain the SASE power spectrum

$$
\begin{aligned}
\frac{d P}{d \omega} \approx \frac{\int d^{2} \bar{x}\left|A_{0}(\overline{\boldsymbol{x}})\right|^{2}}{|C|^{2}} e^{2 \mu_{l} \bar{z}} \frac{\rho \gamma_{0} m c^{2}}{2 \pi} \int d^{2} \bar{x}^{\prime}\left|A_{0}\left(\overline{\boldsymbol{x}}^{\prime}\right)\right|^{2} \int d^{2} \bar{p} U\left(\overline{\boldsymbol{p}}^{2}+\bar{k}_{3}^{2}\left(\overline{\boldsymbol{x}}^{\prime}\right)^{2}\right) \\
\times \int d \bar{\eta} \boldsymbol{V}(\bar{\eta}) \frac{1}{\left[\bar{\eta}-\left(\overline{\boldsymbol{p}}^{2}+\bar{k}^{2} \overline{\boldsymbol{x}}^{2}\right) / 2-\mu_{R}\right]^{2}+\mu_{I}^{2}} .
\end{aligned}
$$


We have taken $f_{0}=U\left(\overline{\boldsymbol{p}}^{2}+\bar{k}_{\beta}^{2} \overline{\boldsymbol{x}}^{2}\right) V(\bar{\eta})$ with

$$
U\left(\bar{p}^{2}+\bar{k}_{3}^{2} \bar{x}^{2}\right)=\frac{1}{2 \pi \bar{k}_{\beta}^{2} \bar{\sigma}_{x}^{2}} \exp \left(-\frac{\bar{p}^{2}+\bar{k}_{\beta}^{2} \bar{x}^{2}}{2 \bar{k}_{\beta}^{2} \bar{\sigma}_{x}^{2}}\right)
$$

where $\bar{\sigma}_{x}=\sigma_{x} \sqrt{2 k_{1} k_{u} \rho}$ is the scaled transverse beam size and is related to the beam emittance $\epsilon=\bar{\sigma}_{x}^{2} \bar{k}_{3} / k_{1}$. If we further assume that the external focusing strength $\bar{k}_{\beta}$ is much larger than the undulator natural focusing $\bar{k}$, which is the case in $x$-ray FELs such as the Linac Coherent Light Source (LCLS) [17], we can neglect $\bar{k}^{2} \overline{\boldsymbol{x}}^{2}$ in comparison with $\overline{\boldsymbol{p}}^{2}$ in Eq. (32) and identify the effective start-up noise power as

$$
\begin{gathered}
\left(\frac{d P}{d \omega}\right)_{S} \approx \frac{\rho \gamma_{0} m c^{2}}{2 \pi \mu_{I}^{2}} \\
\int d^{2} \bar{x}^{\prime}\left|A_{0}\left(\overline{\boldsymbol{x}}^{\prime}\right)\right|^{2} \exp \left(-\frac{\overline{\boldsymbol{x}}^{2}}{2 \bar{\sigma}_{x}^{2}}\right) \int \frac{d^{2} \bar{p}}{2 \pi \bar{k}_{\beta}^{2} \bar{\sigma}_{x}^{2}} \exp \left(-\frac{\overline{\boldsymbol{p}}^{2}}{2 \bar{k}_{\beta}^{2} \bar{\sigma}_{x}^{2}}\right) \\
\times \int d \bar{\eta} V(\bar{\eta})\left[1+\left(\frac{\bar{\eta}-\overline{\boldsymbol{p}}^{2} / 2-\mu_{R}}{\mu_{I}}\right)^{2}\right]^{-1}
\end{gathered}
$$

Equation (34) provides a similar phase-space convolution as the spontaneous undulator radiation when the effects of electron angular spread is taken into account [18], except that the spectrum response function is a Lorentzian instead of the undulator spectrum $S_{u}\left(\frac{\bar{\eta}-\bar{p}^{2} / 2-\bar{v}}{2 \mu_{I}}\right)$ at one field gain length. Identifying $\mu_{I}$ as the bandwidth of $\bar{\eta}$ (or $\bar{\nu}$ ) as in Sec. 4 and $\sqrt{\mu_{I}}$ as the rms angular spread of the fundamental mode (or $2 L_{g}=\left(k_{u} \mu_{I} \rho\right)^{-1}$ as the Rayleigh length), we may interpret the effective start-up noise as the phase-space convolution of the spontaneous undulator radiation in the first field gain length with the coherent fundamental laser mode.

For numerical computation, we approximate $A_{0}(\bar{x})=\exp \left(-w|\bar{x}|^{2} / \bar{\sigma}_{x}^{2}\right)$, where $w=w_{R}+w_{I}$ is a complex number characterizing the Gaussian fundamental mode. Equation (32) can be written as

$$
\frac{d P}{d \omega} \approx g_{A}^{3 d} g_{S}^{3 d} \frac{\rho \% m c^{2}}{2 \pi} e^{2 \mu_{I} \bar{z}}
$$


where

$$
\begin{aligned}
& g_{A}^{3 d} \approx\left|1-4 i w \int_{-\infty}^{0} d \tau_{1} \int_{-\infty}^{0} d \tau_{2} \frac{\left(\tau_{1}+\tau_{2}\right) \exp \left[-\frac{\bar{\sigma}_{\eta}^{2}}{2}\left(\tau_{1}+\tau_{2}\right)^{2}-i \mu_{0}\left(\tau_{1}+\tau_{2}\right)\right]}{\left[1+i \bar{k}_{\beta}^{2} \bar{\sigma}_{x}^{2}\left(\tau_{1}+\tau_{2}\right)\right]\left[1+4 w+i \bar{k}^{2} \bar{\sigma}_{x}^{2}\left(\tau_{1}+\tau_{2}\right)\right]}\right|^{-2}, \\
& g_{S}^{3 d} \approx \frac{4|w|^{2}}{w_{R}} \int_{-\infty}^{0} d \tau_{1} \int_{-\infty}^{0} d \tau_{2} \frac{\exp \left[-\frac{\tilde{\sigma}_{n}^{2}}{2}\left(\tau_{1}-\tau_{2}\right)^{2}-i\left(\mu_{0} \tau_{1}-\mu_{0}^{*} \tau_{2}\right)\right]}{\left[1+i \bar{k}_{\beta}^{2} \bar{\sigma}_{x}^{2}\left(\tau_{1}-\tau_{2}\right)\right]\left[1+4 w_{R}+i \bar{k}^{2} \bar{\sigma}_{x}^{2}\left(\tau_{1}-\tau_{2}\right)\right]} .
\end{aligned}
$$

For example, using the current LCLS design parameter [17], we have $\bar{\sigma}_{r}=2.8$, $\bar{\sigma}_{\eta}=0.45, \bar{k}=0.07$, and $\bar{k}_{\beta}=0.29$. The fundamental guided mode has a complex growth rate $\mu_{0}=-1.2+0.42 i$ and a mode profile determined by $w=0.64-0.50 i$ at the optimal detuning $\bar{\nu}_{0}=-1.0$ [10]. Hence we obtain $g_{A}^{3 d} \approx 0.3$ and $g_{S}^{3 d}=2.3$, both larger than the values with vanishing energy spread and emittance.

\section{Conclusion}

In summary, an analytical solution to the initial value problem in a high-gain FEL is obtained via Van Kampen's method, including the effects of beam energy spread and emittance, as well as the diffraction and guiding of the radiation. In the absence of any external signal, the effective start-up noise is shown to be the phase-space convolution of the spontaneous undulator radiation in one field gain length with the coherent fundamental laser mode. Compared with the $1-\mathrm{D}$, cold beam solution $[1,2]$, the input coupling coefficient for CA and SASE is larger with finite energy spread, and the effective start-up noise also appears to be larger with beam energy spread and emittance mainly due to the increase in gain length. Fluctuation in initial electron velocities (due to beam energy spread and angular spread) do not seem to contribute to any additional start-up noise. 
This work was supported by the U. S. Department of Energy, Office of Basic Energy Sciences, under Contract No. W-31-109-ENG-38.

\section{References}

[1] K.-J. Kim, Nucl. Instr. Meth. A 250, 396 (1986).

[2] J.-M. Wang and L.-H. Yu, ibid, 484 (1986).

[3] K.-J. Kim, Phys. Rev. Lett. 57, 1871 (1986).

[4] K.-J. Kim, unpublished notes.

[5] S. Krinsky and L.-H. Yu, Phys. Rev. A 35, 3406 (1987).

[6] L.-H. Yu and S. Krinsky, Nucl. Instr. Meth. A 285, 119 (1989).

[7] M. Xie, presented at the American Physical Society April Meeting, Long Beach, 2000 , and in these proceedings.

[8] R. Bonifacio, C. Pellegrini, and L.M. Narducci, Opt. Comm. 50, 373 (1984).

[9] Y.L. Klimontovich, Sov. Phys. JETP 6, 753 (1958); see also S. Ichimaru, Basic Principles of Plasma Physics, W. A. Benjamin, Inc., London, 1973.

[10] Z. Huang and K.-J. Kim, submitted to Phys. Rev. E, 2000.

[11] N.G. Van Kampen, Physica (Utrecht) 21, 949 (1951); K.M. Case, Ann. Phys. (N.Y.) 7,349 (1959).

[12] L.H. Yu et al., Phys. Rev. E 51, 813 (1995).

[13] L.-H. Yu, S. Krinsky and R.L. Gluckstern, Phys. Rev. Lett. 64, 3011 (1990).

[14] M. Xie, Nucl. Instr. Meth. A 445, 59 (2000); ibid, 67 (2000).

[15] B.M. Kincaid, J. Appl. Phys. 482684 (1977).

[16] E.L. Saldin. E.A. Schneidmiller and M.V. Yurkov, Nucl. Instr. Meth. A 313 555 (1992); see also The Physics of Free Electron Lasers, Springer, Berlin, 1999.

[17] Linac Coherent Light Source Design Study Report, SLAC-R-521, 1998.

[18] See, for example, K.-J. Kim, in AIP Conference Proceedings 184, M. Month and M. Dienes. eds.. 585 (1989). 


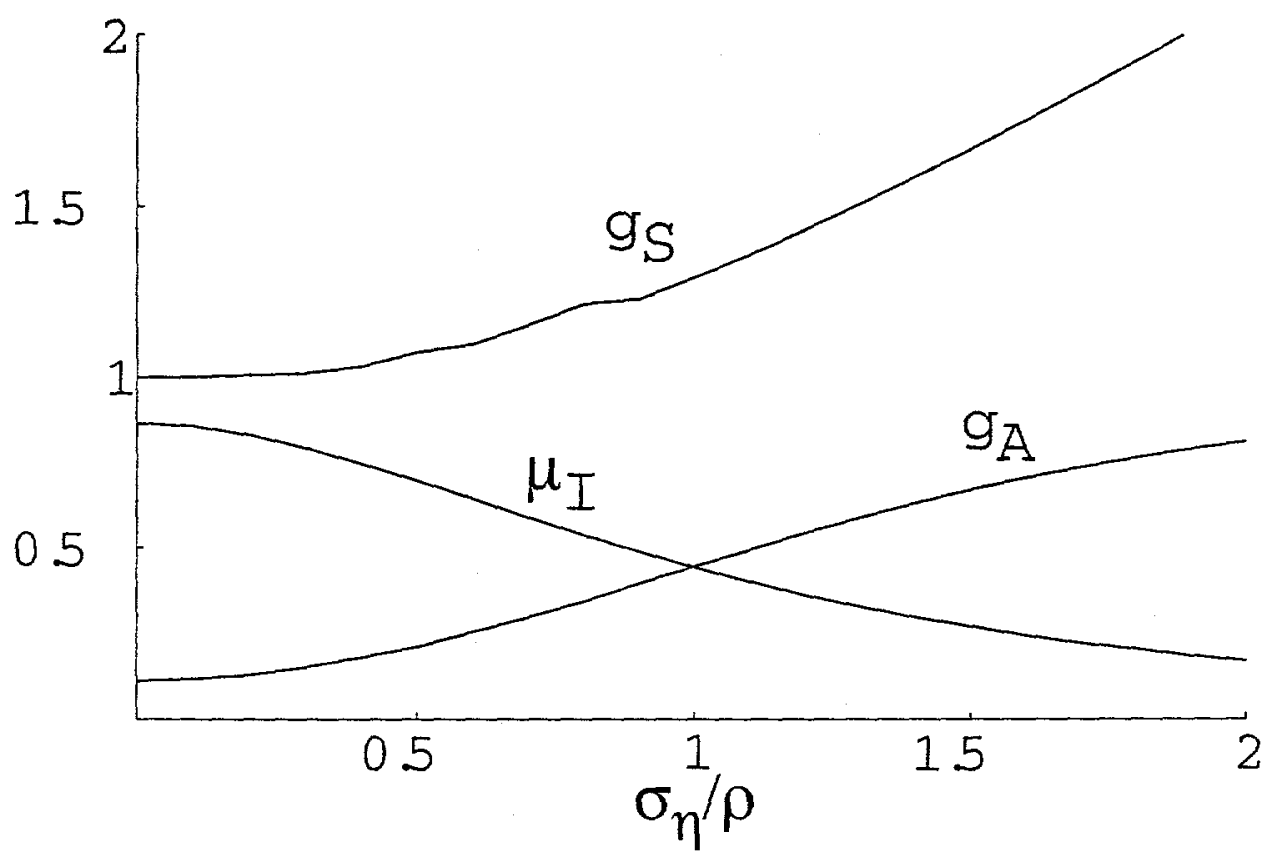

Fig. 1. The behavior of $\mu_{I}, g_{A}$ and $g_{S}$ as functions of the rms energy spread $\bar{\sigma}_{\eta}=\sigma_{\eta} / \rho$ for a Gaussian energy distribution. 